\title{
Characterization of Pectin Nanocoatings at Polystyrene and Titanium Surfaces
}

\author{
Katarzyna Gurzawska ${ }^{1,2^{*}}$, Kai Dirscherl ${ }^{3}$, Yu Yihua $^{4}$, Inge Byg ${ }^{5}$, Bodil Jørgensen ${ }^{5}$, Rikke Svava ${ }^{5}$, \\ Martin W. Nielsen ${ }^{6}$, Niklas R. Jørgensen ${ }^{1}$, Klaus Gotfredsen ${ }^{2}$ \\ ${ }^{1}$ Research Center for Ageing and Osteoporosis, Departments of Medicine and Diagnostics, Copenhagen University Hospital Glostrup, \\ Glostrup, Denmark; ${ }^{2}$ Institute of Odontology, Faculty of Health and Medical Sciences, University of Copenhagen, Copenhagen, \\ Denmark; ${ }^{3}$ Dansk Fundamental Metrologi A/S, Lyngby, Denmark; ${ }^{4}$ Microtechnology and Surface Analysis, Danish Technological \\ Institute, Taastrup, Denmark; ${ }^{5}$ Department of Plant and Environment Sciences, Faculty of Science, University of Copenhagen, \\ Frederiksberg, Denmark; ${ }^{6}$ Department of Systems Biology, Technical University of Denmark, Lyngby, Denmark. \\ Email: *kagu@sund.ku.dk
}

Received June $20^{\text {th }}, 2013$; revised July $19^{\text {th }}, 2013$; accepted August $6^{\text {th }}, 2013$

Copyright (C) 2013 Katarzyna Gurzawska et al. This is an open access article distributed under the Creative Commons Attribution License, which permits unrestricted use, distribution, and reproduction in any medium, provided the original work is properly cited.

\begin{abstract}
The titanium implant surface plays a crucial role for implant incorporation into bone. A new strategy to improve implant integration in a bone is to develop surface nanocoatings with plant-derived polysaccharides able to increase adhesion of bone cells to the implant surface. The aim of the present study was to physically characterize and compare polystyrene and titanium surfaces nanocoated with different Rhamnogalacturonan-Is (RG-I) and to visualize RG-I nanocoatings. RG-Is from potato and apple were coated on aminated surfaces of polystyrene, titianium discs and titanium implants. To characterize, compare and visualize the surface nanocoatings measurements of contact angle measurements and surface roughness with atomic force microscopy, scanning electron microscopy, and confocal microscopy was performed. We found that, both unmodified and enzymatic modified RG-Is influenced surface wettability, without any major effect on surface roughness (Sa, Sdr). Furthermore, we demonstrated that it is possible to visualize the pectin RG-Is molecules and even the nanocoatings on titanium surfaces, which have not been presented before. The comparison between polystyrene and titanium surface showed that the used material affected the physical properties of non-coated and coated surfaces. RG-Is should be considered as a candidate for new materials as organic nanocoatings for biomaterials in order to improve bone healing.
\end{abstract}

Keywords: Surface Properties; Titanium; Polystyrene; Rhamnogalacturonan-I; Osseointegration

\section{Introduction}

The implant surface plays a crucial role for implant incorporation into the bone and implant surface modifications which are continuously developed in attempts to enhance and accelerate bone formation at the implant surface [1-5]. The development has been approached by chemically and physically modifications of the surface [1,3-8]. The first concept focuses on incorporating inorganic and/or organic molecules at the surface whereas the second focuses on changing surface properties including the surface topography $[3,9]$. The chemical and physical surface modification can be performed at different levels $[1,3,6]$. From a biological point of view, the osseointegration process takes place at the cellular level,

\footnotetext{
"Corresponding author.
}

and therefore especially micro and nanoscale investigations have great importance for developing new surfaces $[4,6]$. It has been demonstrated that nanoscale modification of titanium implants affects surface properties, such as hydrophilicity, biochemical bonding capacity and roughness, which influence cell behaviour on the surface such as adhesion, proliferation and differentiation of cells as well as the mineralization of the extracellular matrix at the implant surfaces [2,4-6,9-12].

The inorganic and organic nanocoatings are continuously developed and tested in vitro and in vivo. For in vitro examination, Tissue Culture Polystyrene Surfaces (TCPS) or titanium discs (Grade 2 or 4 ) are most frequently used [9], whereas for in vivo experiments titanium implant surfaces of Grade 4 titanium are the most frequently used $[1,12]$. To obtain the best conformity be- 
tween in vitro and in vivo studies and thereby the best prerequisite for interpretation of in vitro results, studies characterizing and comparing how nanocoatings influence the surfaces are important.

It has been shown that both polystyrene and titanium surfaces coated with Rhamnogalacturonan-I (RG-I) affect osteoblast cell responses. By enhancing osteoblast attachment, proliferation and mineralization compared to uncoated surfaces [9-11,13-17]. The biological mechanism when nanocoating RG-Is onto polystyrene and titanium surfaces is however still not fully understood. The positive biological effect might be connected to RG-Is' structure, but also to the change of surface properties caused by RG-Is nanocoatings.

The aim of the presented study was to physically characterize and compare polystyrene and titanium surfaces nanocoated with different Rhamnogalacturonan-Is (RGIs) and to visualize the RG-I nanocoatings.

\section{Materials and Methods}

In order to characterize the RG-Is pectin nanocoating, three different types of material surfaces were used: 1) Tissue Culture Polystyrene Plates (TCPS), (Nunc, Roskilde, Denmark) with a diameter of $60 \mathrm{~mm}$; 2) titanium discs (Ti discs) with a diameter of $13 \mathrm{~mm}$ (Astra Tech, Mölndal, Sweden); 3) titanium implants (Ti implants) with a diameter of $3.5 \mathrm{~mm}$ and a length of $8 \mathrm{~mm}$ (ANKYLOS, Dentsply, Konstanz, Germany). We included 7 different surfaces. Five were coated with RG-Is and two were noncoated (Table 1).

\subsection{Coating Procedure}

To obtain a covalent bonding between the surfaces and

Table 1. Surfaces and nanocoatings used in the study.

\begin{tabular}{|c|c|c|c|c|}
\hline & & \multicolumn{3}{|c|}{ Material of the surface } \\
\hline \multicolumn{2}{|c|}{ Surface } & \multirow{2}{*}{$\begin{array}{c}\text { Polystyrene } \\
\text { TCPS }\end{array}$} & \multicolumn{2}{|c|}{ Titanium } \\
\hline & & & Ti discs & Ti implants \\
\hline \multirow{2}{*}{ Non-coated } & Untreated & + & + & + \\
\hline & Aminated & + & + & + \\
\hline \multirow{5}{*}{ Coated } & PU & + & + & + \\
\hline & PA & + & + & + \\
\hline & PG & + & + & - \\
\hline & PAG & + & + & - \\
\hline & $\mathrm{AU}$ & + & + & + \\
\hline
\end{tabular}

+ used in study; - not used in the study; TCPS: Tissue Culture Polystyrene Surface Plates; Ti discs: Titanium discs, Ti implants: Titanium implants; PU: Potato unmodified RG-I, PA: Potato dearabinanated RG-I; PG: Potato degalactanated RG-I; PAG: Potato dearabinanated degalactanated RG-I; AU: Apple unmodified RG-I, RG-I: Rhamnogalacturonan-I. the RG-I coatings all coated surfaces were aminated. Surface amination of Tissue Culture Polystyrene Plates (TCPS), titanium (Ti) discs and implants was performed by plasma polymerization of allylamine following the procedure described by Morra et al. [16] The RG-Is were covalently coupled via reaction between the carboxyl groups present in GalA of the RG-I backbone and the primary amino groups on the surface [16].

\subsection{Physical Characterization}

Physical characterization of TCPS and titanium surfaces was performed using contact angle measurements for wettability, scanning electron microscopy (SEM) for visualization of the surface texture and atomic force microscope (AFM) for surface roughness measurements and visualization of the nanocoating. The measurements were performed on 3 samples $(n=3)$ of the TCPS, the Ti discs and the $\mathrm{Ti}$ implants and at four different areas $(\mathrm{m}=$ 4) for each sample. The measurement areas of TCPS and Ti discs were selected randomly on the surface and at the Ti implants the measurements were performed at the top, valley and flanks according to recommendation by Wennerberg (2010) [18].

1) Contact angles (sessile angle) were measured using a KRUSS Drop Shape Analysis System, DSA10-Mk2 (Kruss GmbH, Hamburg, Germany). A water droplet (2.5 $\mu \mathrm{L}$ on TCPS plates and Ti discs surface and $1 \mu \mathrm{L}$ on crest of Ti implants surface) was dropped on the surface and recorded photographically. Contact angles were measured in the recorded image by using drop shape analysis software, Scientific Drop Shape Analysis Software, DSA 1, Version 1.70 (Kruss GmbH, Hamburg, Germany) and a curve fitting method (Tangent Method-1).

2) Scanning Electron Microscopy (SEM) was performed with a ZEISS Ultra 55 scanning electron microscope (Carl Zeiss NTS GmbH, Oberkochen, Germany) operating at $3 \mathrm{kV}$ and $20 \mathrm{kV}$ in the secondary electron imaging mode. Images were collected at $1 \mathrm{~K}, 5 \mathrm{~K}, 10 \mathrm{~K}$, $20 \mathrm{~K}, 30 \mathrm{~K}, 35 \mathrm{~K}, 40 \mathrm{~K}$ or $50 \mathrm{~K}$.

3) Surface roughness was measured with a metrologic Atomic Force Microscope (AFM) DIM3100m (Bruker AXS Inc., Fitchburg, WI, USA). The AFM had a scan volume of $70 \mu \mathrm{m} \times 70 \mu \mathrm{m} \times 7 \mu \mathrm{m}$ and intermittent scan mode (tapping mode) was used to minimize interaction force between tip and sample. The applied tip had a specified tip radius of $10 \mathrm{~nm}$. The samples were scanned with a scan rate of $0.1 \mathrm{~Hz}$ to minimize scan artefacts in the image profiles. For the analysis of the roughness measurements, the scanned images were pre-processed with a first order lateral plane fit. This corrects for the residual sample tilt and no further filtering was applied. The parameters selected for analysis of surface roughness were Sa and Sdr using an area of $20 \mu \mathrm{m} \times 20 \mu \mathrm{m}$. [3] The same conditions and equipment were used for visulaiza- 
tion of RG-I nanocoatings on titanium implant surfaces, but the measurements were performed with $1 \times 1 \mu \mathrm{m}$ scan area.

\subsection{Visualization of RG-Is Nanocoatings}

1) For visualization of RG-Is structures Atomic Force Microscopy (AFM) imaging was performed using a multimode AFM (Bruker AXS Inc., Fitchburg, WI, USA) with a Nano $\mathrm{V}$ controller. RG-Is from potato unmodified (PU), potato dearabinanated (PA), potato degalactanated $(\mathrm{PG})$, potato dearabinanated degalactanated $(\mathrm{PAG})$ and apple unmodified (AU) were dispersed into mili-Q water to a concentration of $1 \mu \mathrm{g} \cdot \mathrm{mL}^{-1}$. Aliquots $\left(10 \mathrm{~mL}^{-1}\right)$ of the diluted RG-Is samples were deposited onto a freshlycleaved molecularly smooth surface [19-21] (Mica surfaces, Sa $\sim 0 \mathrm{~nm}$, Tedpella, Redding, CA, USA) and allowed to dry under ambient conditions before imaging by AFM in air [22]. The samples were scanned in intermittent "tapping" mode with commercial tips "SSS-NCH" from NanoSensors with a tip radius of $2 \mathrm{~nm}$. Scannings was performed in ambient conditions. The scan rate was set to $0.5 \mathrm{~Hz}$ to limit the scan speed to $1 \mu \mathrm{m} / \mathrm{s}$ for a typical sized image of $1 \mu \mathrm{m} \times 1 \mu \mathrm{m}$. Using the image processing software SPIP (Image Metrology, Hørsholm, Denmark), the data was line-wise tilt-corrected with a first order fit restricted to the data points of the Mica surface only. This allows accurate height measurements relative to the flat Mica reference surface with measurement uncertainties below $1 \mathrm{~nm}$.

2) Immunofluorescence labeling and confocal microscopy was performed on four implants, one non-coated aminated Ti implant and three coated Ti implants with (PU, PA, AU). The implants were placed in polystyrene 24-well plate (Nunc, Roskilde, Denmark) in separated wells and blocked for $15 \mathrm{~min}$ with $1 \mathrm{ml} /$ well of $5 \%$ skimmed milk from Applichem (Darmstadt, Germany) (5\% solution of fat-free milk powder in phosphate buffered saline (PBS), $\mathrm{pH}$ 7.2). Skimmed milk was removed from the well and $1 \mathrm{ml} /$ well of anti- $(1 \rightarrow 4)-\beta$-galactan LM5 (IgG2c) (PlantProbes, Leeds, UK) diluted 1:10 in $5 \%$ skimmed milk was added and placed on a shaker for $2 \mathrm{~h}$. LM5 was removed from all wells and all implants were washed with 5\% skimmed milk 3 times (after adding the milk to the wells, the plate was placed on a shaker for $5 \mathrm{~min}$ ). Secondary antibody, goat anti-rat $\operatorname{IgG}$ for LM5 linked to FITC (fluorescein isothiocyanate) from Sigma-Aldrich (Brøndby, Denmark) was diluted 1:200 in $5 \%$ of skimmed milk and applied $1 \mathrm{ml} /$ well. The plate was covered by aluminum foil and placed on shaker for 2 hours. Subsequently. implants were washed three times. $1 \mathrm{ml}$ of PBS was added to each well in order to store the implants until examination by confocal microscopy at 4 degrees. Confocal images were done with a Leica TCSSP5 II confocal laser scanning microscope (Leica Mi- crosystems, Exton, PA, USA) with PL Fluotar 10/x0.30 DRY objective with the same setting and conditions as described in our previous work [23].

\subsection{Osteoblast Cell Culture}

SaOS-2 osteoblast-like cells were grown on Ti implants under the same conditions as described in our previous studies [23]. The cell morphology observations were done with a Leica TCS-SP5 II confocal laser scanning microscope (Leica Microsystems, Exton, PA, USA).

\subsection{Statistical Analysis}

Descriptive statistics were calculated as mean values and standard errors of the mean. Results of surface analysis experiments were analysed using ANOVA tests and Bonferroni corrections for multiple comparisons using SPSS 11.5 software. A significance level of 0.05 was used throughout the study. More sensitive statistics between uncoated (untreated and aminated) samples and coated (PU, PA, PG, PAG, AU) samples, as well as between different type of surfaces (TCPS, Ti discs and Ti implants) was applied.

\section{Results}

\subsection{Physical Characterization}

\subsubsection{Contact Angle}

When the contact angles of the 3 surfaces (TCPS, Ti discs, Ti implants) were compared only significant differences were found between TCPS and Ti disc surfaces $(p=0.004)$. The highest SEM values were found for the titanium implants (Figure 1).

When all 3 different surfaces were compiled the con-

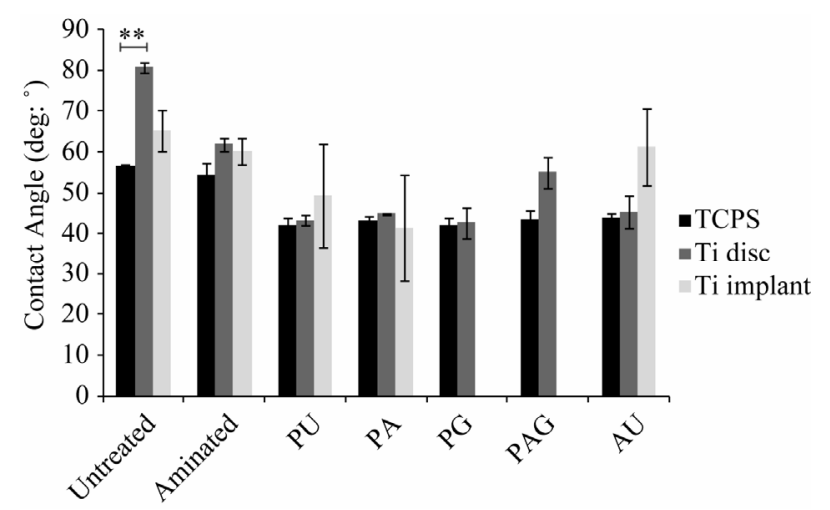

Figure 1. The contact angle (sessile angle) measurements results represent mean contact angle values and standard error of the mean (mean \pm SEM). Uncoated surfaces: untreated, aminated and coated surfaces: PU (potato unmodified), PA (potato dearabinanated), PG (potato degalactanated), PAG (potato dearabinanated and degalactanated) and AU (apple unmodified) of TCPS, Ti discs and Ti implants surfaces. Ti: titanium. 
tact angle measurements demonstrated that nanocoating with RG-Is (PU, PA, PG, PAG, AU), gave significantly $(\mathrm{p}=0.006)$ lower contact angles compared to the noncoated control surfaces (untreated and aminated) (Figure 1). RG-I surfaces coated with PU $(p=0.02)$, PA ( $p=$ $0.01)$ and $P G(p=0.02)$ had significantly lower contact angles compare to the untreated surfaces.

When analyzing differences between non-coated and coated TCPS surfaces significant differences were found for all coatings $(p<0.001)$. For the Ti discs significant differences were found for all coatings compared to controls, except for PAG compared to aminated surfaces. For the titanium implants no significant differences between coated and non-coated surfaces (Figure 1).

\subsubsection{Surface Roughness}

The results for surface roughness ( $\mathrm{Sa}, \mathrm{Sdr})$ measurements are shown in Figures 2(a) and (b). In general the Ti implants were significantly more rough than the Ti discs, which were significantly more rough than the TCPS plates.

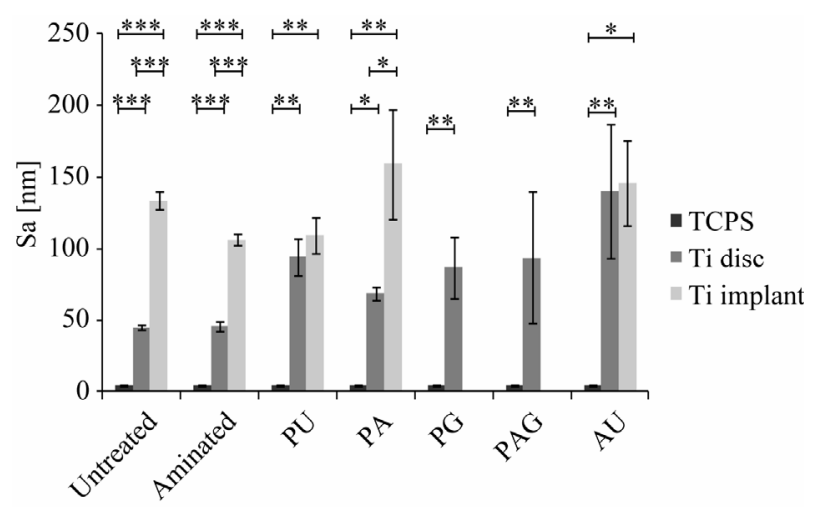

(a)

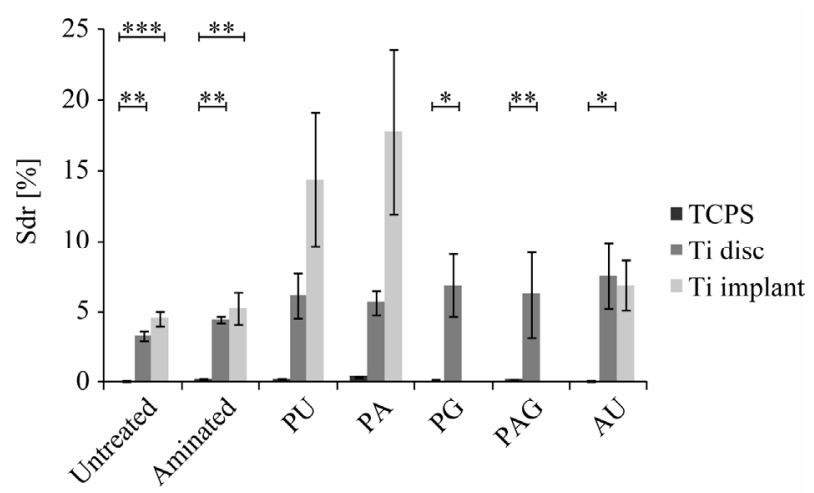

(b)

Figure 2. Surface roughness measured with AFM and represented by amplitude parameter (Sa) (a) and hybrid parameter (Sdr); (b) (means \pm SEM). Untreated, aminated, PU (potato unmodified), PA (potato dearabinanated), PG (potato degalactanated), PAG (potato dearabinanated and degalactanated) and AU (apple unmodified) of TCPS, Ti discs and Ti implants surfaces. Ti: titanium.
When the 3 different surfaces were compiled no significant differences in $\mathrm{Sa}(\mathrm{p}=0.97)$ and $\mathrm{Sdr}(\mathrm{p}=0.86)$ values were found between coated and non-coated surfaces.

When analyzing differences for the TCPS surfaces, significant differences in Sa value were found for nanocating with PU $(p=0.02)$, PA $(p=0.003)$, PG $(p=$ $0.001)$, PAG $(p=0.001)$ and AU $(p=0.001)$ compared to untreated TCPS surface, but not to the aminated surfaces. When analyzing differences in Sa value of noncoated and coated surfaces of Ti discs the only significant difference $(p=0.011)$ was found between AU coated and untreated Ti discs $(\mathrm{p}=0.016)$ and aminated Ti discs $(p=0.018)$. When analyzing differences in Sdr values of uncoated and coated surfaces the only significant difference was found on TCPS surfaces $(p=0.013)$, between PU coated TCPS and non-coated TCPS surfaces $(p=0.026)$. No significant differences in $\mathrm{Sa}$ and $\mathrm{Sdr}$ values were found between coated and non-coated $\mathrm{Ti}$ implants.

\subsubsection{Scanning Electron Microscopy (SEM)}

The SEM images of TCPS, Ti disc and Ti implants (Figure 3) showed differences in surface texture. On the surface of titanium discs and implants, the texture pattern (machined surface) from the manufacturing process could be observed on the SEM images.

\subsection{Visualization of Pectin Nanocoatings}

\subsubsection{RG-Is Structure Observed with AFM}

The structure of potato RG-Is (scan area of $1 \mu \mathrm{m} \times 1 \mu \mathrm{m}$ ) is demonstrated at Figure 4. The enzymatic modification reduces the size of the pectin molecule. More than 70 measurements were conducted for each type for various individual pectins. The height of the potato RG-Is are shown in Figure 5. The results are sorted in decreasing order for better visualization of the height differences. After enzymatic modification of the arabinan and galactan side chains, the height of PU decreased significantly by approximately $3 \mathrm{~nm}$ on average (Figure 5).

\subsubsection{RG-Is Nanocoating on Titanium Implant Surfaces Visualized with AFM}

A representative 3D image of PA RG-I nanocoating is shown in Figure 6 with linear structures and a heterogenous distribution of the RG-I molecules on the surface of the Ti implants.

\subsubsection{Immunofluorescence Labeling and Confocal Microscopy}

The confocal images showed presence of RG-Is nanocoating on the coated titanium implant surface compared to control aminated titanium implant surface (Figure 7). 

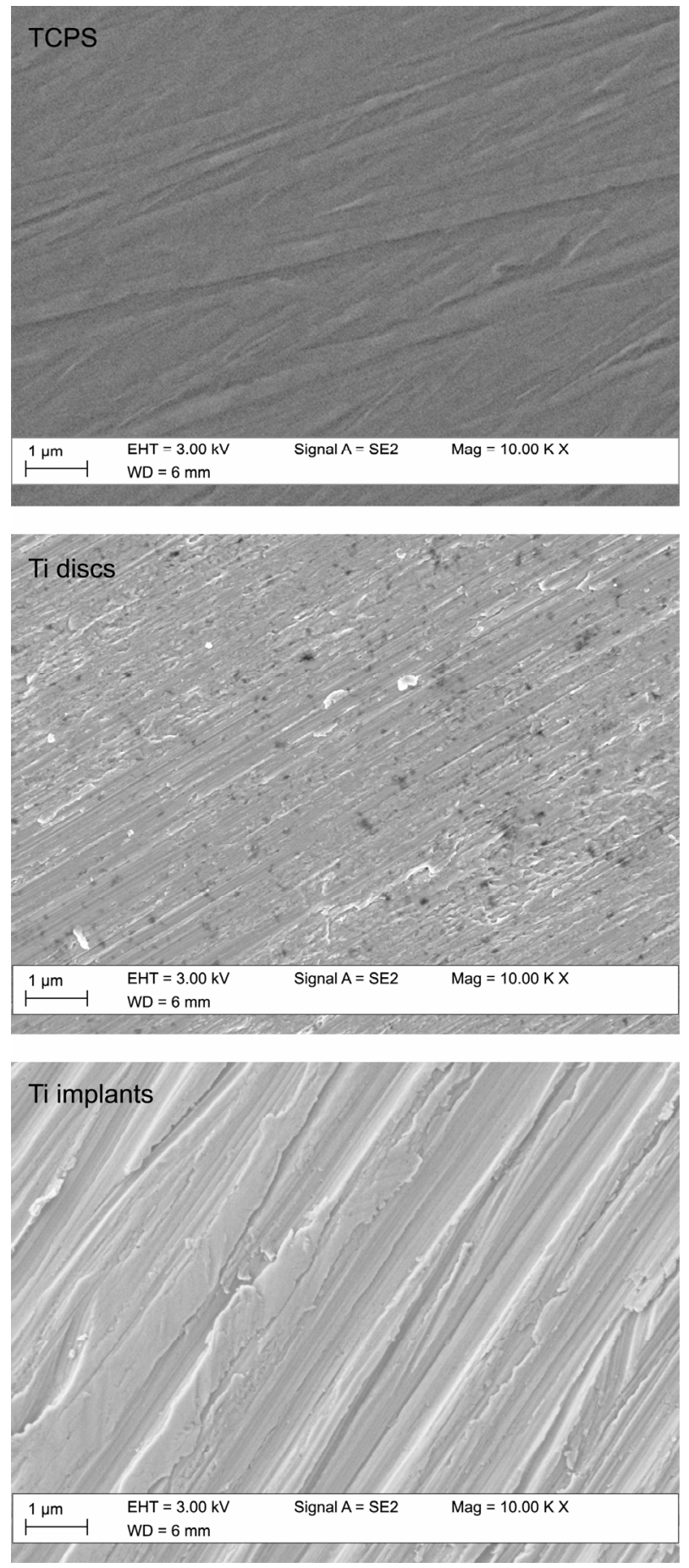

Figure 3. Representative images of untreated control surface of TCPS, Ti disc and Ti implant performed with SEM at 3 kV. TCPS: Tissue Polystyrene Plate, Ti: titanium, WD: working distance, EHT: the high voltage, SE2: type of detector.

\subsection{Osteoblast Cell Culture}

The confocal images from the Ti implants cultured with $\mathrm{SaO} 2$ cells (Figure 8) showed spread morphology of the osteoblast-like cells on RG-Is nanocoated titanium im- plants as well as on the control aminated Ti implant.

\section{Discussion}

In this work, we assessed the effect on physical properties of nanocoatings with potato and apple RG-Is to polystyrene and titanium surfaces. We found that native (PU, AU) and modified RG-Is (PA, PG, PAG) influenced surface wettability, without any major influence on surface roughness (Sa, Sdr). Furthermore, we demonstrated that it is possible to visualize the pectin molecules and even the nanocoatings on titanium surfaces, which have not been presented before. The comparison between polystyrene and titanium surfaces showed that both materials became more hydrophilic after nanocoating and that the RG-I molecules did not have any major effect on the surface roughness.

In accordance with the present study, a number of other studies have demonstrated that RG-Is nanocoatings influenced the physical properties of polystyrene $[11,13$, $16,17]$ and titanium surfaces $[10,14,15,23]$. According to findings by Morra et al. (2004), the difference in wettability between RG-I coated and non-coated polystyrene surfaces is caused by changes in the chemical composition after coating with RG-Is [16]. The same results were presented in a work by Kokonnen et al. (2006), where the authors proposed that the RG-Is' side chains produce a hydrated gel-like surface [11]. Our previous work also demonstrated that by changing the chemical composition of the surface with RG-Is nanocoatings the wettability of the polystyrene surface was affected and the surface became more hydrophilic compared to non-coated control surfaces [14]. The same results were observed on titanium surfaces showing that coating with RG-Is gave rise to smaller contact angles compared to the controls i.e. more hydrophilic surfaces $[10,14,23]$. In our study the comparison of different surface TCPS, Ti discs and Ti implants coated with RG-Is confirmed these findings. However at the titanium implants there was a limited access to measurements, which may explain the lack of significant difference in the contact angle between coated and non-coated surfaces. The increased hydrophilicity obtained by nanocoating with RG-Is seemed to be similar at the TCPS, Ti discs and titanium implants. The fact that RG-I coatings created a more hydrophilic surface can have a positive impact on osseointegration, as hydrophilic surfaces are more suitable for interaction with biological fluids, cells and tissues than a more hydrophobic surface [24]. In our study, a significant decrease in the contact angle on the surface coated with PU, PA and PG was shown, compared to the untreated control surface. Therefore, these RG-Is should be considered for further in vitro and in vivo studies.

The change in wettability of the surface has been reported to be related not only to chemical modification but 
Potato unmodified

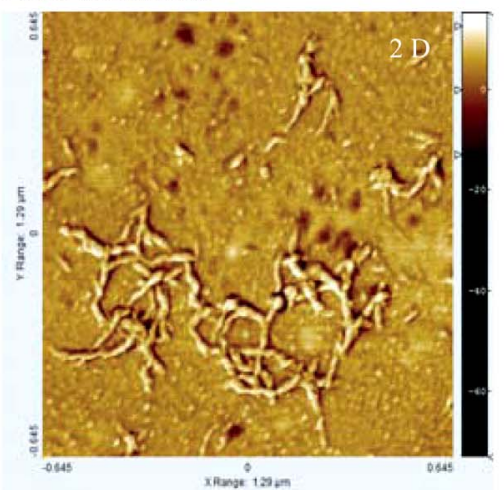

xhmog 120

Potato dearabinanated

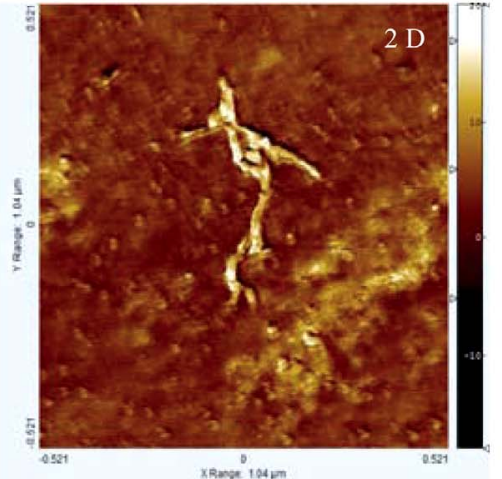

Potato degalactanated

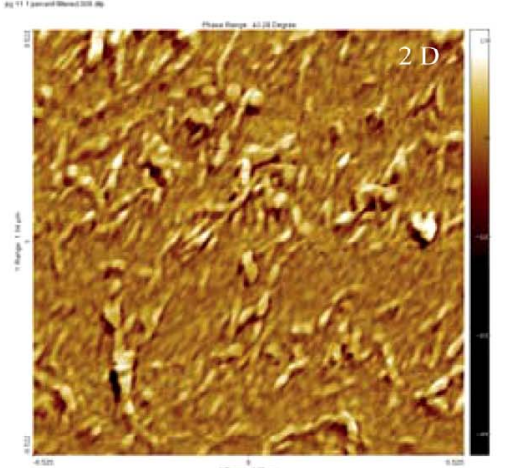

Potato dearabinanated degalactanated

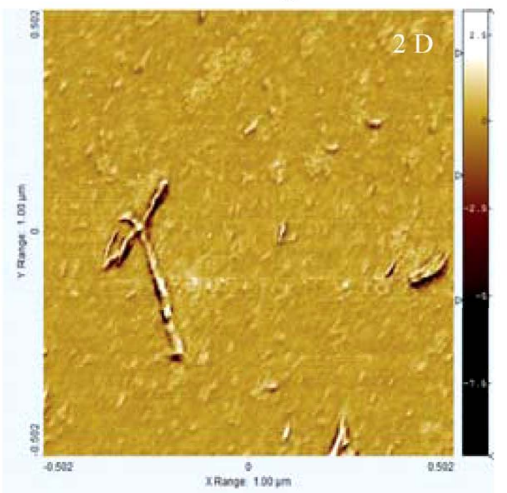

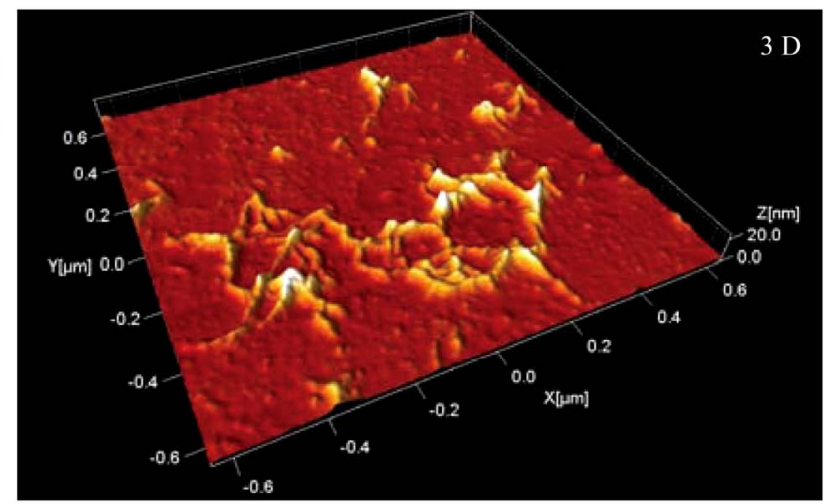

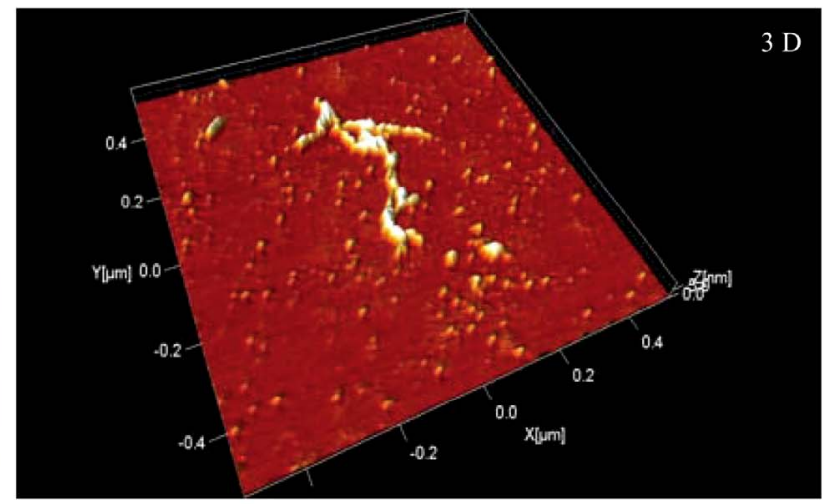

$3 \mathrm{D}$
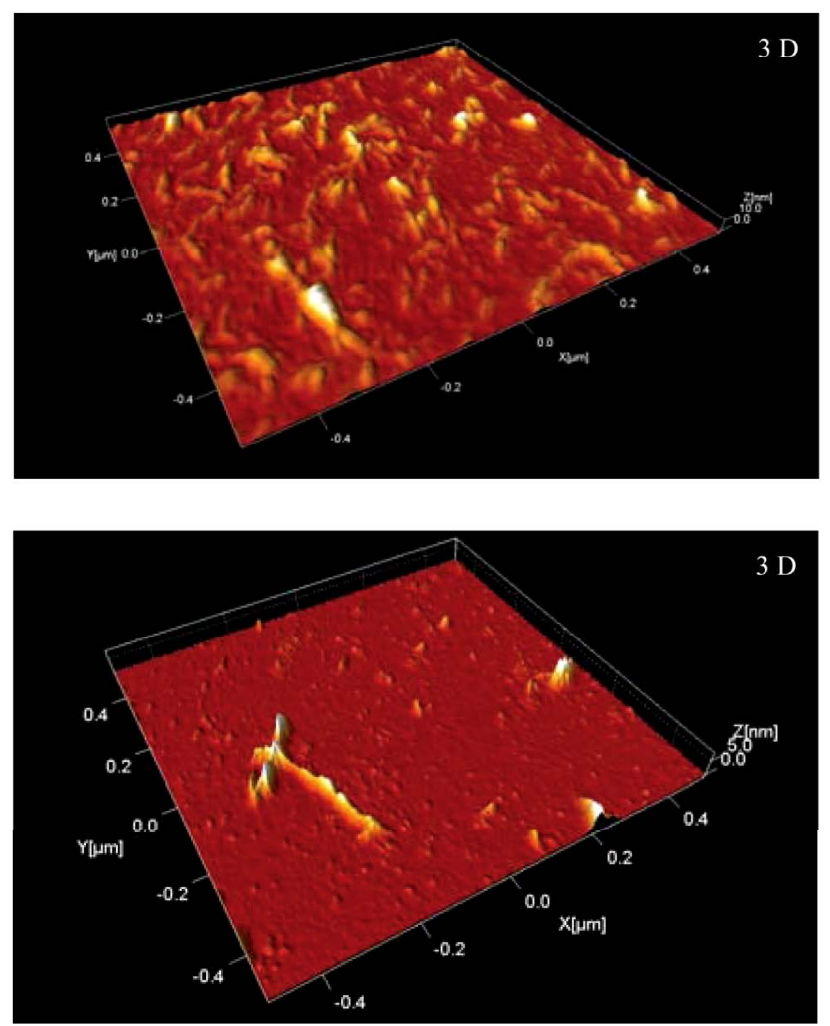

Figure 4. Representative images (2D and 3D) of RG-Is structure: potato unmodified (PU), potato dearabinanated (PA), potato degalactanated (PG), potato dearabinanated degalactanated (PAG) on mica surface measured with a Multimode AFM 1 $\mu \mathrm{m} \times 1 \mu \mathrm{m}$. 


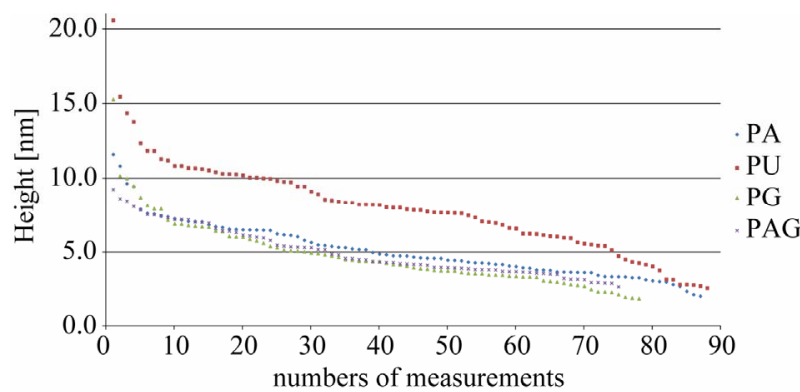

Figure 5. Distribution plot of height measurements in $\mathbf{n m}$ of RG-Is structure of PU, PA, PG, PAG performed with AFM on Mica surface with $1 \mu \mathrm{m} \times 1 \mu \mathrm{m}$ magnification. Potato unmodified (PU), potato dearabinanated (PA) and potato degalactanated (PG) and potato dearabinanated degalactanated (PAG).

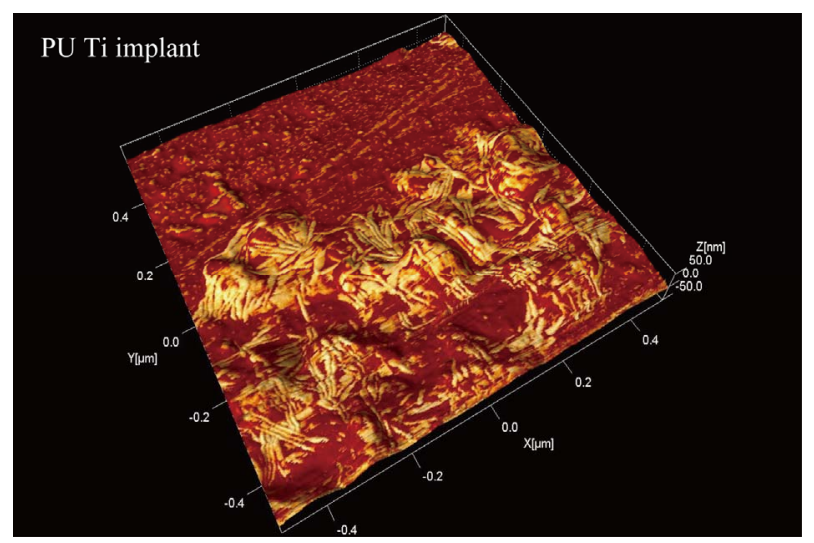

Figure 6. Representative image (3D) of RG-I nanocoating on aminated titanium implant surface coated with potato dearabinanated (PA aminated Ti implant), magnification $1 \times 1$ $\mu \mathrm{m}$, measured using AFM with intermittent "Tapping mode".
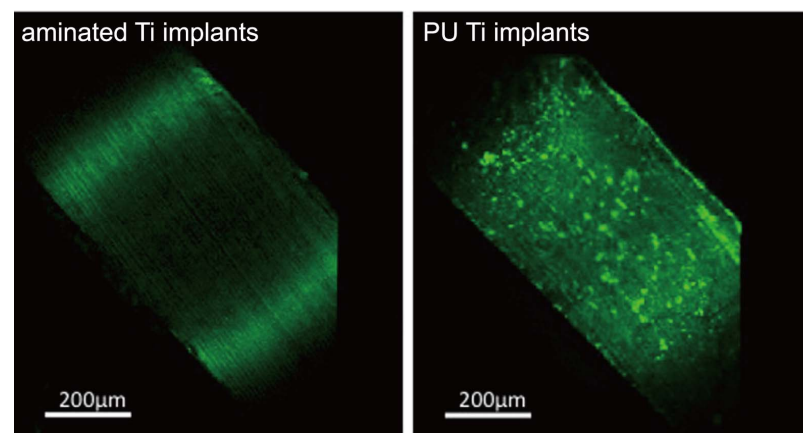

Figure 7. Representative confocal images of RG-Is nanocoating visualised with immunofluorescence labeling by primary antibody, anti $-(1 \rightarrow 4)$ - $\beta$-galactan LM5 (IgG2c) and secondary antibody, goat anti-rat IgG for LM5 linked to FITC. Control: aminated Ti implant, Coated: PU Ti implant; Ti: titanium, PU: potato unmodified.

also to the topographical changes of the surface $[25,26]$. Our surface roughness results showed that the RG-Is used for nanocoating in general did not significantly affect the roughness of the examined surfaces, which is in-
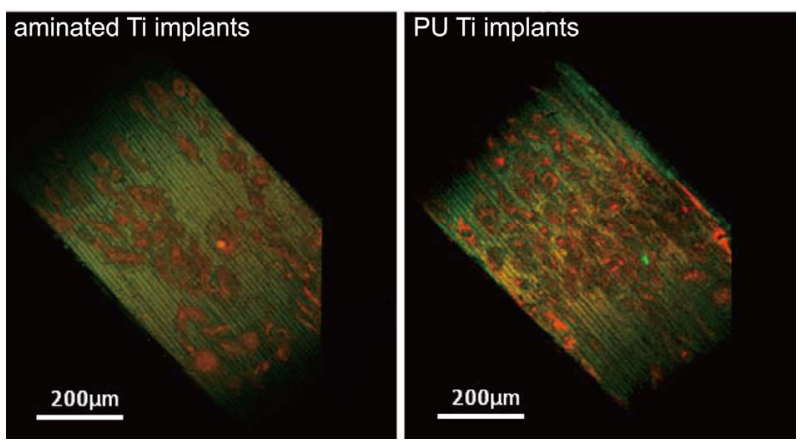

Figure 8. Representative confocal images of SaOS-2 cells stained with Vybrant Cell-Labeling Solutions and cultured on Ti implants surface nanocoated with RG-Is. Control: aminated Ti implant, Coated: PU Ti implant; Ti: titanium, PU: potato unmodified.

accordance with findings from Morra et al. (2004) [16]. The reason for different results of surface roughness ( $\mathrm{Sa}$, Sdr) on polystyrene and titanium surface, when each of the surface groups was compared, can be explained by differences in surface texture, illustrated by the SEM images. It has also to be noticed that the surface roughness was measured by AFM on a $20 \mu \mathrm{m} \times 20 \mu \mathrm{m}$ square area as recommended for a non quantitative overview of the nanotopography [27]. Higher magnification would probably show difference between nanocoated and noncoated surfaces as the RG-I molecules used for coating are around $100 \mathrm{~nm}$ in size. Thus, our measurements performed with AFM on a $1 \mu \mathrm{m} \times 1 \mu \mathrm{m}$ area clearly visualized the RG-Is nanocoating also on the titanium implant surface. Nanocoatings of that size have not previously been demonstrated. The visualization of the nanocoating may be important for characterizing the physical properties of the nanocoated surface. The structure, as well as the distribution and the topography of the nanocoating, may play an important role in cell adhesion, as the nanocoating can mimic the extracellular matrix (ECM) [28]. In the present study, we also visualized RG-Is nanocoating on titanium implants by immunofluorescence staining using the primary LM5 antibody, which specifically binds to galactan side chains. By using the AFM technique with atomically smooth surfaces, we were able to visualize and analyze the height and length of RG-Is from potato. The length of the individual molecule was in the range of $100 \mathrm{~nm}$, which is in agreement with other studies [22]. The height measurements showed a decrease in the height of modified RG-Is (PA, PG, PAG) compared to unmodified RG-Is (PU), which showed that enzymatic modification changed the RG-Is structure. This corresponds to our previous findings, demonstrating that enzymatic treatment of RG-Is decreases the amount of galactan and arabinan in modified RG-Is compared to unmodified RG-Is (PU). [14] Our height measurements of modified RG-Is have not allowed us to distinguish 
between dearabinanated, degalactananated and debranched structures and therefore more detailed investigations with AFM in "liquid cell" (AFM imaging in liquids) are necessary [29].

The osteblast-like cells (SaOS2) grown on Ti implants were spread on the surface, however the morphology, cell viability and proliferation studies remain to be performed to examine the osteoblast behavior on titanium implant surface coated with RG-Is. On the other hand, our previous studies showed a significant increase in cell viability and matrix mineralization of the same type of cells $(\mathrm{SaOS} 2)$ on polystyrene and titanium discs surfaces coated with RG-Is containing higher amounts of galactose compared to controls [14]. Recent in vitro studies [10,11,14-16] showed that the nanocoating of RG-I with high amounts of galactose enhanced osteoblast spreading and growth, in contrast to the nanocoating of RG-I with high amounts of arabinose, which leads to aggregation and decreased proliferation [11,13,14,16,17]. This finding suggests that linear 1.4-linked galactans are important for osteoblast adhesion, and that high content of arabinose can shield the galactans, and thus prohibiting their interaction with osteoblasts [30]. It has previously been shown that Galectin-3 binds specifically to galactose residues [31]. As the osteoblastic cells contain Galectin-3, it could therefore be speculated that osteoblast interaction with the RG-I galactans is mediated through Galectin-3. In addition, titanium surfaces coated with RG-Is have been shown to positively influence cell adhesion, morphology, proliferation and mineralization $[10,15,23]$. The positive cell response on plant-derived molecules, especially RG-I with high amount of galactose, opens new direction in the development of organic nanocoatings for biomaterials in order to improve bone healing.

\section{Acknowledgements}

The authors thank Prof. Erik Fink Eriksen from Aarhus Amtssygehus in Denmark for the gift of the SaOS-2 cell line and Marco Morra for the aminated TCPS plates, Ti discs and Ti implants.

\section{REFERENCES}

[1] T. Albrektsson and A. Wennerberg, "Oral Implant Surfaces: Part 1-Review Focusing on Topographic and Chemical Properties of Different Surfaces and in Vivo Responses to Them," The International Journal of Prosthodontics, Vol. 17, No. 5, 2004, pp. 536-543.

[2] T. Albrektsson and A. Wennerberg, "Oral Implant Surfaces: Part 2-Review Focusing on Clinical Knowledge of Different Surfaces," The International Journal of Prosthodontics, Vol. 17, No. 5, 2004, pp. 544-564.

[3] D. M. Dohan Ehrenfest, P. G. Coelho, B. S. Kang, Y. T. Sul and T. Albrektsson, "Classification of Osseointegrated Implant Surfaces: Materials, Chemistry and To- pography," Trends in Biotechnology, Vol. 28, No. 4, 2010, pp. 198-206.

http://dx.doi.org/10.1016/j.tibtech.2009.12.003

[4] G. Mendonca, D. B. Mendonca, F. J. Aragao and L. F. Cooper, "Advancing Dental Implant Surface Technology-From Micron- to Nanotopography," Biomaterials, Vol. 29, No. 28, 2008, pp. 3822-3835. http://dx.doi.org/10.1016/j.biomaterials.2008.05.012

[5] M. Morra, "Biochemical Modification of Titanium Surfaces: Peptides and ECM Proteins," European Cells and Materials Journal, Vol. 12, 2006, pp. 1-15.

[6] L. T. de Jonge, S. C. Leeuwenburgh, J. G. Wolke and J. A. Jansen, "Organic-Inorganic Surface Modifications for Titanium Implant Surfaces," Pharmaceutical Research, Vol. 25, No. 10, 2008, pp. 2357-2369.

http://dx.doi.org/10.1007/s11095-008-9617-0

[7] G. L. Le, A. Soueidan, P. Layrolle and Y. Amouriq, "Surface Treatments of Titanium Dental Implants for Rapid Osseointegration," Dental Materials, Vol. 23, No. 7, 2007, pp. 844-854. http://dx.doi.org/10.1016/j.dental.2006.06.025

[8] R. Junker, A. Dimakis, M. Thoneick and J. A. Jansen, "Effects of Implant Surface Coatings and Composition on Bone Integration: A Systematic Review," Clinical Oral Implants Research, Vol. 20, Suppl. 4, 2009, pp. 185-206. http://dx.doi.org/10.1111/j.1600-0501.2009.01777.x

[9] K. Gurzawska, R. Svava, N. R. Jørgensen and K. Gotfredsen, "Nanocoating of Titanium Implant Surfaces with Organic Molecules. Polysaccharides Including Glycosaminoglycans," Journal of Biomedical Nanotechnology, Vol. 8, No. 6, 2012, pp. 1012-1024. http://dx.doi.org/10.1166/jbn.2012.1457

[10] H. Kokkonen, C. Cassinelli, R. Verhoef, M. Morra, H. A. Schols and J. Tuukkanen, "Differentiation of Osteoblasts on Pectin-Coated Titanium," Biomacromolecules, Vol. 9, No. 9, 2008, pp. 2369-2376. http://dx.doi.org/10.1021/bm800356b

[11] H. E. Kokkonen, J. M. Ilvesaro, M. Morra, H. A. Schols and J. Tuukkanen, "Effect of Modified Pectin Molecules on the Growth of Bone Cells," Biomacromolecules, Vol. 8, No. 2, 2007, pp. 509-515. http://dx.doi.org/10.1021/bm060614h

[12] A. Wennerberg and T. Albrektsson, "On Implant Surfaces: A Review of Current Knowledge and Opinions," The International Journal of Oral \& Maxillofacial Implants, Vol. 25, No. 1, 2010, pp. 63-74.

[13] C. Bussy, R. Verhoef, A. Haeger, M. Morra, J. L. Duval, P. Vigneron, et al., "Modulating in Vitro Bone Cell and Macrophage Behavior by Immobilized Enzymatically Tailored Pectins," Journal of Biomedical Materials Research Part A, Vol. 86A, No. 3, 2008, pp. 597-606. http://dx.doi.org/10.1002/jbm.a.31729

[14] K. Gurzawska, R. Svava, S. Syberg, Y. Yihua, K. B. Haugshoj, I. Damager, et al., "Effect of Nanocoating with Rhamnogalacturonan-I on Surface Properties and Osteoblasts Response," Journal of Biomedical Materials Research Part A, Vol. 100, No. 3, 2012, pp. 654-664. http://dx.doi.org/10.1002/jbm.a.33311

[15] H. Kokkonen, H. Niiranen, H. A. Schols, M. Morra, F. 
Stenback and J. Tuukkanen, "Pectin-Coated Titanium Implants Are Well-Tolerated in Vivo," Journal of Biomedical Materials Research Part A, Vol. 93, No. 4, 2010, pp. 1404-1409.

[16] M. Morra, C. Cassinelli, G. Cascardo, M. D. Nagel, C. Della Volpe, S. Siboni, et al., "Effects on Interfacial Properties and Cell Adhesion of Surface Modification by Pectic Hairy Regions," Biomacromolecules, Vol. 5, No. 6, 2004, pp. 2094-2104. http://dx.doi.org/10.1021/bm049834q

[17] M. D. Nagel, R. Verhoef, H. Schols, M. Morra, J. P. Knox, G. Ceccone, et al., "Enzymatically-Tailored Pectins Differentially Influence the Morphology, Adhesion, Cell Cycle Progression and Survival of Fibroblasts," Biochimica et Biophysica Acta, Vol. 1780, No. 7, 2008, pp. 995-1003.

[18] A. Wennerberg and T. Albrektsson, "Suggested GuideLines for the Topographic Evaluation of Implant Surfaces," The International Journal of Oral \& Maxillofacial Implants, Vol. 15, No. 3, 2000, pp. 331-344.

[19] L. Cheng, P. Fenter, K. L. Nagy, M. L. Schlegel and N. C. Sturchio, "Molecular-Scale Density Oscillations in Water Adjacent to a Mica Surface," Physical Review Letters, Vol. 87, No. 15, 2001, Article ID: 156103. http://dx.doi.org/10.1103/PhysRevLett.87.156103

[20] T. Matsuura, H. Tanaka, T. Matsumoto and T. Kawai, “Atomic Force Microscopic Observation of Escherichia coli Ribosomes in Solution," Bioscience, Biotechnology, and Biochemistry, Vol. 70, No. 1, 2006, pp. 300-302. http://dx.doi.org/10.1271/bbb.70.300

[21] C. H. Lui, L. Liu, K. F. Mak, G. W. Flynn, T. F. Heinz, "Ultraflat Graphene," Nature, Vol. 462, No. 7271, 2009, pp. 339-341. http://dx.doi.org/10.1038/nature08569

[22] K. T. Inngjerdingen, T. R. Patel, X. Chen, L. Kenne, S. Allen, G. A. Morris, et al., "Immunological and Structural Properties of a Pectic Polymer from Glinus opposetifolius," Glycobiology, Vol. 17, No. 12, 2007, pp. 12991310. http://dx.doi.org/10.1093/glycob/cwm088

[23] K. A. Gurzawska, R. Svava, Y. Yihau Dr., K. B. Haugshøj, K. Dirscherl, S. B. Levery, I. Byg, I. Damager, B. Jørgensen, N. R. Jørgensen and K. Gotfredsen, "Osteoblastic Response to Pectin Nanocoating of Titanium Surface," Submitted, under Review.

[24] S. Tajima, J. S. Chu, S. Li and K. Komvopoulos, "Dif- ferential Regulation of Endothelial Cell Adhesion, Spreading, and Cytoskeleton on Low-Density Polyethylene by Nanotopography and Surface Chemistry Modification Induced by Argon Plasma Treatment," Journal of Biomedical Materials Research Part A, Vol. 84, No. 3, 2008, pp. 828-836. http://dx.doi.org/10.1002/jbm.a.31539

[25] F. Rupp, L. Scheideler, N. Olshanska, M. de Wild, M. Wieland and J. Geis-Gerstorfer, "Enhancing Surface Free Energy and Hydrophilicity through Chemical Modification of Microstructured Titanium Implant Surfaces," Journal of Biomedical Materials Research Part A, Vol. 76A, No. 2, 2006, pp. 323-334. http://dx.doi.org/10.1002/jbm.a.30518

[26] S. Tosatti, M. Textor and N. D. Spencer, "Self-Assembled Monolayer of Dodecyl and Hydroxy-Dodecyl Phosphate at Smooth and Rough Titanium and Titanium Oxide Surfaces," Langmuir, Vol. 18, No. 9, 2002, pp 3537 3548 .

[27] A. Bagno and B. C. Di, "Surface Treatments and Roughness Properties of Ti-Based Biomaterials," Journal of Materials Science: Materials in Medicine, Vol. 15, No. 9, 2004, pp. 935-949. http://dx.doi.org/10.1023/B:JMSM.0000042679.28493.7f

[28] F. Munarin, S. G. Guerreiro, M. A. Grellier, M. C. Tanzi, M. A. Barbosa, P. Petrini, et al., "Pectin-Based Injectable Biomaterials for Bone Tissue Engineering," Biomacromolecules, Vol. 12, No. 3, 2011, pp. 568-577. http://dx.doi.org/10.1021/bm101110x

[29] V. J. Morris, A. Gromer, A. R. Kirby, R. J. M. Bongaerts and A. Patrick Gunning, "Using AFM and Force SpecTroscopy to Determine Pectin Structure and (bio) Functionality," Food Hydrocolloids, Vol. 25, No. 2, 2011, pp. 230-237. http://dx.doi.org/10.1016/j.foodhyd.2009.11.015

[30] H. Kokkonen, R. Verhoef, K. Kauppinen, V. Muhonen, B. Jorgensen, I. Damager, et al., "Affecting Osteoblastic Responses with in Vivo Engineered Potato Pectin Fragments," Journal of Biomedical Materials Research Part A, Vol. 100A, No. 1, 2012, pp. 111-119. http://dx.doi.org/10.1002/jbm.a.33240

[31] A. P. Gunning, R. J. Bongaerts and V. J. Morris, "Recognition of Galactan Components of Pectin by Galectin-3," The FASEB Journal, Vol. 23, No. 2, 2009, pp. 415-424. http://dx.doi.org/10.1096/fj.08-106617 